\title{
Variabilidad de la frecuencia cardiaca en pacientes con cardiopatía chagásica
}

\section{Heart rate variability in patients with Chagas' heart disease}

\author{
José Ricardo Corredor-Matus ${ }^{1}$; Fernando Riveros-Sanabria²; Laura Tatiana Corredor-Mesa ${ }^{3}$
}

\begin{abstract}
1MVZ, M.Sc., Universidad de los Llanos, Escuela Ciencias Animales, Grupo de Investigación MACRYPT. Villavicencio - Meta, Colombia; e-mail: jcorredor@ unillanos.edu.co; (D) https://orcid.org/0000-0002-2434-7928

${ }^{2}$ Ing. Electrónico. M.Sc., CPhD, Universidad de los Llanos, Escuela de Ingeniería, Grupo de Investigación MACRYPT. Villavicencio - Meta, Colombia; e-mail: friveros@unillanos.edu.co; (i) https://orcid.org/0000-0001-8948-6956

${ }^{3}$ Médico Cirujano. Western Desert Nganampa Walytja Palyantjaku Tjutaku Aboriginal Corporation, Alice Springs-Australia; e-mail: laticorme@hotmail.com; (D) https://orcid.org/0000-0003-3495-0306
\end{abstract}

Cómo citar: Corredor-Matus, J.R.; Riveros-Sanabria, F.; Corredor-Mesa, L.T. 2020. Variabilidad de la frecuencia cardiaca en pacientes con cardiopatía chagásica. Rev. U.D.C.A Act. \& Div. Cient. 23(2):e1197. http://doi.org/10.31910/rudca.v23.n2.2020.1197

Artículo de acceso abierto publicado por Revista U.D.C.A Actualidad \& Divulgación Científica, bajo una licencia Creative Commons CC BY-NC 4.0

Publicación oficial de la Universidad de Ciencias Aplicadas y Ambientales U.D.C.A, Institución de Educación Superior Acreditada de Alta Calidad por el Ministerio de Educación Nacional.

Recibido: Abril 1 de 2019 Aceptado: Noviembre 12 de 2020 Editado por: Ingeborg Zenner de Polanía

\section{RESUMEN}

Se estima la prevalencia por Trypanosoma cruqi de 436.000 habitantes en Colombia, con casos anuales de 5.250, en población expuesta de 4.792.000 habitantes. La variabilidad de la frecuencia cardiaca (VFC) es la variabilidad en el tiempo, de un latido cardiaco, medido en un análisis de periodo temporal determinado. Su análisis permite determinar el equilibrio simpático-vagal de manera indirecta. Este estudio analizó las variables temporales y No lineales, en 19 pacientes Chagásicos y 19 controles, utilizándose un polígrafo de alta resolución y el software Kubios. La variable Desviación Estandar del Intervalo RR (SDRR) en la población control arrojó un promedio

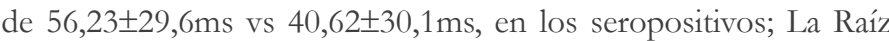
Cuadrada del Promedio de la Suma de las Diferencias al Cuadrado de todos los intervalos Adyacentes (RMSSD) fue de 34,31 $\pm 21,01 \mathrm{~ms}$ y $31,94 \pm 37,33 \mathrm{~ms}$, para controles y Chagas, respectivamente. El número de los intervalos RR consecutivos, que difieren en más de

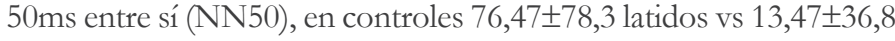
para seropositivos, que correspondió con el porcentaje de NN50

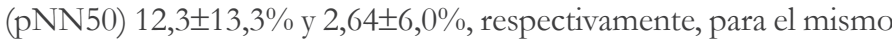
orden de los grupos. Valores de Entropía Aproximada (ApEn) fueron $1,249 \pm 0,134$, para controles y $0,959 \pm 0,325$, para seropositivos y para

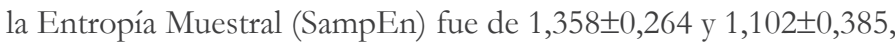
para controles y chagásicos, respectivamente. Se encontró mayor irregularidad de HRV en controles, que es reflejo de un mejor estado de salud.

Palabras clave: Chagas; Frecuencia cardiaca; Entropía; Nervio vago; Variable temporal. 


\section{ABSTRACT}

The prevalence by Trypanosoma cruzi of 436,000 inhabitants in Colombia is estimated, with annual cases of 5,250, in an exposed population of 4,792,000 inhabitants. Heart rate variability (HRV) is the time interval of a beat in a given time analysis. Its analysis allows to determine the sympathetic-vagal balance indirectly. This study analyzed the temporal and non-linear variables in 19 Chagasic patients and 19 controls, using a high-resolution polygraph and the Kubios software. The variable Standard Deviation of the RR Interval (SDRR) in the control population showed an average of $56.23 \pm 29.6 \mathrm{~ms}$ vs $40.62 \pm 30.1 \mathrm{~ms}$ in the seropositive; The Square Root of the Sum of the Square Differences of all Adjacent intervals (RMSSD) was $34.31 \pm 21.01 \mathrm{~ms}$ and $31.94 \pm 37.33 \mathrm{~ms}$ for controls and Chagas respectively. The number of consecutive RR intervals that differ by more than $50 \mathrm{~ms}$ from each other (NN50) in controls $76.47 \pm 78.3$ beats vs $13.47 \pm 36.8$ for seropositives corresponding to the percentage of NN50 (pNN50) $12.3 \pm 13.3 \%$ and $2.64 \pm 6.0 \%$, respectively for the same order of the groups. Approximate Entropy (ApEn) values were 1,249 $\pm 0,134$ for controls and 0,959 $\pm 0,325$ for seropositive, and for Sample Entropy (SampEn) it was 1,358 $\pm 0,264$ and 1,102 $\pm 0,385$ for controls and chagasics respectively. Greater irregularity of HRV was found in controls, which reflects a better state of health.

Keywords: Chagas; Heartbeat; Entropy; Vagus nerve; Temporal variable.

\section{INTRODUCCIÓN}

Se estima que, en América Latina, entre 6 y 7 millones de personas están infectadas por la enfermedad de Chagas, de las cuales, alrededor de un 30\% desarrollará síntomas de enfermedad crónica, en especial, la cardiopatía, provocando alrededor de 12.000 muertes por año (PAHO/WHO, 2017). Se calcula una prevalencia de la infección por Trypanosoma cru₹i, agente etiológico de esta enfermedad, de 436.000 habitantes en Colombia, en donde el número de nuevos casos anuales de transmisión vectorial es de 5.250; la población expuesta en zona endémica es de 4.792.000 (Instituto Nacional de Salud y Organización Panamericana de la Salud, INS/OPS, 2009). Casanare y Meta son dos de los departamentos más afectados (Guhl, 1998). La fase crónica de la enfermedad, se caracteriza por una leve parasitemia y lesiones en corazón y tubo digestivo (Gimenez et al. 2003).

Un estudio realizado por la clínica fundación Shaio, en 120 pacientes con diagnóstico de enfermedad de Chagas (Mora et al. 1998), reportó que los hallazgos electrocardiográficos más notables fueron: bloqueo completo de rama derecha $(40 \%)$, bloqueo aurículo-ventricular de segundo y tercer grado $(29,2 \%)$, disfunción del nodo sinusal $(28,3 \%)$, taquicardia ventricular $(23 \%)$, fibrilación auricular $(19 \%)$, hemibloqueo anterior izquierdo (17,2\%), flutter auricular $(3,3 \%)$ y bloqueo de rama izquierda (3,3\%). La enfermedad, también genera disfunción del sistema nervioso autónomo, relacionado con el incremento del tono simpático y denervación cardiovagal, que se pueden cuantificar, a través de métodos de medición de la variabilidad de la frecuencia cardiaca (VFC) (Tundo et al. 2005).
La VFC es un parámetro definido como la variabilidad en el tiempo, de un latido cardiaco, medido en un análisis de periodo temporal determinado (Sánchez et al. 2013; Rasmussen et al. 2014). Su determinación está basada en la medición de ondas $\mathrm{R}$ consecutivas y el cálculo en ms del tiempo, entre ellas (Rodas et al. 2008; Buzzano et al. 2012; Khor et al. 2014; Bogucki \& Nosczczyk-Nowak. 2015). El estudio de la VFC permite determinar el equilibrio simpático-vagal de una manera indirecta (Belerenian et al. 2001; Álvarez \& Cruz, 2011; Buzzano et al. 2012; Merejo et al. 2018; Fortes et al. 2018).

La VFC, se evalúa a través de parámetros de Dominio Temporal, de frecuencia y medidas No Lineales (Rodas et al. 2008; Rasmussen et al. 2014; Merejo et al. 2018; Fortes et al. 2018). Los de Tiempo resultan de la medición de los intervalos RR, que se afectan por la actividad del sistema nervioso autónomo e incluyen: Desviación estándar del Intervalo RR (SDRR), Raíz Cuadrada del Promedio de la Suma de las diferencias al cuadrado de todos los intervalos adyacentes (RMSSD), número de los intervalos RR consecutivos, que difieren en más de $50 \mathrm{~ms}$ entre sí (NN50) y porcentaje de NN50 (pNN50) (Rodas et al. 2008; Rasmussen et al. 2014; Fortes et al. 2018).

Los parámetros no lineales son una medida cualitativa de la VFC, miden la estructura o complejidad de las series de intervalos RR (Bar-Yam, 1997). Incluye la entropía aproximada (ApEn) y la entropía muestral (SampEn), la cual, es menos dependiente de la longitud de series de tiempo (Richman \& Moorman, 2000). Los parámetros no lineales han sido usados para analizar y clasificar la VFC (Sady \& Ribeiro, 2016; Fortes et al. 2018).

La VFC es una herramienta poco utilizada en la consulta médica, por lo que se desaprovecha su información, que puede ser de gran utilidad, como ayuda para el diagnóstico médico de la enfermedad de Chagas. Dado que la cardiopatía chagásica genera disfunción de la actividad del sistema nervioso autónomo y esta disfunción se puede valorar con el estudio de la VFC, el análisis de esta variable podría ser utilizada para identificar pacientes con afección autonómica, diagnosticados con enfermedad de Chagas y para quienes la valoración de la VFC podría ser utilizada como alerta temprana.

Por lo descrito anteriormente, se planteó como objetivo realizar un análisis de la VFC en los dominios de tiempo y No lineales en pacientes diagnosticados con enfermedad de Chagas y compararlos con población control, a fin de detectar diferencias significativas, que puedan ayudar a orientar el pronóstico de esta enfermedad en pacientes con cardiopatía crónica, que pueda ser concomitante con el padecimiento de la enfermedad.

\section{MATERIALES Y MÉTODOS}

Se ejecutó en el laboratorio de fisiología de la Universidad de los Llanos (44’30" N, 73³5’7" W), localizado a 4 kilómetros de Villavicencio, vereda Barcelona.

Como población experimental, se recurrió a la base de datos de la Secretaría Departamental de Salud del Meta, para tener acceso 
a pacientes diagnosticados por esta entidad con enfermedad de Chagas, de acuerdo con sus protocolos. De esta base de datos, se contactaron 50 pacientes entre hombres y mujeres, quienes residían en diferentes municipios del departamento del Meta; se incluyeron aquellos pacientes que habitaban en el área urbana y rural de la ciudad de Villavicencio, 19 de ellos, cinco mujeres y 14 hombres, cuyo promedio de edad fue de 52 años. Los individuos control correspondieron a 19 individuos, 4 mujeres y 15 hombres, cuyo promedio de edad fue de 32 años, quienes manifestaron no padecer ninguna enfermedad en el momento de realizar los registros electrocardiográficos. Los pacientes seropositivos tienen mayor edad, dado que la fase crónica de la enfermedad, cuando afecta el corazón, se desarrolla y diagnostica años después de la infestación del paciente, cuando este asiste a la consulta por cardiopatía.

Los pacientes incluidos en el estudio fueron trasladados de manera individual a las instalaciones del laboratorio de Fisiología de la Escuela de Ciencias Animales de la Universidad de los Llanos. Se explicó el objetivo del estudio y el procedimiento. Firmado el consentimiento informado, el paciente se ubicó en un cubículo independiente, temperatura controlada de $24^{\circ} \mathrm{C}$ y humedad relativa del 65\%; se contó con la presencia de una asistente de enfermería, siguiendo las recomendaciones del comité de Bioética institucional. Se obtuvo el registro electrocardiográfico de la derivada DII, la cual, se utilizó como única para este estudio.

Para la obtención del registro en DII, se utilizó un polígrafo marca ADinstruments de 8 canales referencia Power Lab 8/30 (Sydney, Australia), con una resolución de 62,5 nanovoltios, escala de +/- 2 $\mathrm{mV}$, ruido de $2,2 \mu \mathrm{V}$, filtro pasa-bajos de $0-50 \mathrm{~Hz}$ y una velocidad de muestreo de 2000 por segundo, dotado de un acondicionador para ECG acoplado a un bioamplificador con puerto DIN, con entrada de tres electrodos. El polígrafo procesa la información capturada con la ayuda del software LabChart Pro para OS X de apple, para el modelo MLS260/8 de ADInstruments. La visualización del registro, se efectuó a través de la conexión del polígrafo, con un computador portátil; debido a la alta resolución solo se utilizó la derivada D II, con un rango de tiempo de muestreo entre 3 y 10 minutos de duración.

Obtenidos cada uno de los registros, se procesaron con la ayuda del software arriba mencionado, que toma toda la información del muestreo y reporta los parámetros electrocardiográficos. Estos reportes, se almacenaron en un procesador portátil y se exportaron al programa Excel. Para la obtención de los parámetros de VFC, la información almacenada en Excel se exportó al software KUBIOS versión 2.1 de University of Eastern Finland, Kuopio (Finlandia), que procesa los datos y reporta los siguientes parámetros de la VFC, utilizados en este estudio: Parámetros de dominio temporal: FC(lpm), SDRR(ms), SDFC, RMSSD(ms), NN50(l), pNN50(\%); parámetros no lineales: ApEn y SampEn. Tanto los parámetros electrocardiográficos reportados por Labchart Pro como los de VFC reportados por Kubios, se organizaron en un archivo Excel para, posteriormente, someterlos al análisis estadístico.

El procesamiento estadístico, se realizó en el programa SPSS Statistics 17.0.2 2009, con el cual, se obtuvo, inicialmente, la estadística descriptiva de los parámetros de VFC de los dos grupos (Seropositivos a Chagas y controles). Posteriormente, se efectuó análisis de varianza de una vía, que incluyó tratamientos y las variables temporales y no lineales de la VFC, para establecer diferencias significativas entre ellos. Para determinar diferencias significativas entre grupos, se corrió una prueba T de muestras independientes. Los resultados, se expresan en promedios $\pm \mathrm{SD}$. Se consideraron diferencias significativas a valores de $\mathrm{P} \leq 0,05$

\section{RESULTADOS Y DISCUSIÓN}

Se obtuvieron valores en el dominio temporal y medidas no lineales, tanto de los pacientes seropositivos a la enfermedad de Chagas (Tabla 1) como de individuos control (Tabla 2).

Variable SDRR: Esta variable en la población control arrojó un promedio de 56,23 $\pm 29,6 \mathrm{~ms}$, mientras que para los seropositivos

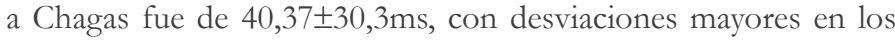
primeros, sin diferencias significativas entre los grupos $(\mathrm{P}=0,09)$. Los valores de RMSSD fueron de 34,32 $\pm 21,01 \mathrm{~ms}$ y $31,94 \pm 37,33 \mathrm{~ms}$ para controles y Chagas, respectivamente, siendo mayor para los pacientes sanos que para los enfermos, sin diferencias significativas $(\mathrm{P}=0,07)$.

La variable SDRR fue la más reducida en los seropositivos a Chagas. Este hallazgo concuerda con el reporte de Gimenez et al. (2003), en cuanto a no encontrar asociación significativa entre variables de VFC entre pacientes controles y chagásicos, pero sí evidencian la prevalencia de altas alteraciones en los parámetros temporales de la VFC en pacientes seropositivos; sin embargo, en este estudio, el $54,1 \%$ de los pacientes chagásicos tuvieron alteraciones significativas de la VFC con respecto a los controles, en los que las alteraciones solo afectaron al 4\% de la población. De igual forma, Mitelman et al. (2015) y Mitelman et al. (2013) reportaron diferencias altamente significativas $(\mathrm{P}<0,0001)$ de VFC entre pacientes chagásicos y controles, en ambos estudios. De la Cruz et al. (2008) establecieron rangos para parámetros de VFC, de modo que SDRR menores a $50 \mathrm{~ms}$ fueron considerados de variabilidad alta; de $50-100 \mathrm{~ms}$, moderada y mayores a $100 \mathrm{~ms}$, baja, lo cual, colocaría a los pacientes chagásicos de este estudio con una variabilidad alta, de acuerdo con esta apreciación. Ortis et al. (2008) reportaron valores de SDRR de individuos sanos, que diferenciaron de acuerdo con su calidad de vida, de $69,78 \pm 27,89 \mathrm{~ms}$ y de $46,0 \pm 16,65 \mathrm{~ms}$, para los de alta $\mathrm{y}$ baja, respectivamente. El último dato es más bajo que el reportado para nuestros controles, pero más elevado que el registrado para los seropositivos de Chagas de este estudio. Ortiz \& Mendoza (2008) reportaron valores para SDRR en un rango entre 30,9$66,8 \mathrm{~ms}$, lo que ubica los datos de este estudio, dentro de ese rango. Ramos et al. (2014) estudiaron el efecto del ejercicio en la VFC de pacientes chagásicos, en comparación con pacientes chagásicos no ejercitados, analizando la variable SDRR y no encontraron diferencias significativas entre los dos grupos, lo cual, se esperaba por el efecto que sobre la VFC tiene el mayor tono vagal. El resultado lo atribuyen a la disfunción del sistema nervioso autónomo y presencia de anticuerpos antimuscarinicos en estos pacientes. 
Tabla 1. Parámetros de HRV en pacientes seropositivos a enfermedad de Chagas.

\begin{tabular}{|l|c|c|c|c|c|c|c|c|}
\hline CÓDIGO & FC (LPM) & SDRR (ms) & SDFC & RMSSD (ms) & NN50 (L) & $\begin{array}{c}\text { pNN50 } \\
\text { (\%) }\end{array}$ & $\begin{array}{c}\text { ENT. APROX } \\
\text { (ApEn) }\end{array}$ & $\begin{array}{c}\text { ENT. } \\
\text { MUESTRAL } \\
\text { (SampEn) }\end{array}$ \\
\hline H001C22 & 87,94 & 34,6 & 4,52 & 13,9 & 1 & 0,1 & 1,19 & 1,24 \\
\hline M002C49 & 78,53 & 39,1 & 4,79 & 24,9 & 8 & 1 & 1,2 & 1,3 \\
\hline M003C58 & 63,89 & 21,7 & 1,47 & 12,8 & 1 & 0,2 & 1,24 & 1,44 \\
\hline H004C53 & 84,06 & 21,4 & 2,58 & 9,7 & 0 & 0 & 1,26 & 1,37 \\
\hline H005C56 & 75,01 & 35,1 & 3,32 & 15,2 & 2 & 0,3 & 1,17 & 1,2 \\
\hline H006C41 & 84,04 & 47,4 & 5,68 & 23,5 & 25 & 3 & 1,33 & 1,44 \\
\hline M007C54 & 68,11 & 26,5 & 2,08 & 10 & 0 & 0 & 1,11 & 1,14 \\
\hline M008C55 & 61,52 & 25,2 & 1,62 & 17,1 & 8 & 1,3 & 1,29 & 1,48 \\
\hline H009C65 & 74,46 & 39,9 & 4,85 & 59,8 & 22 & 2,6 & 0,76 & 0,66 \\
\hline H010C62 & 67,25 & 35,6 & 3,96 & 26,3 & 4 & 0,6 & 1,12 & 1,17 \\
\hline H011C48 & 67,11 & 148,9 & 16,85 & 145,1 & 162 & 25,1 & 0,82 & 0,67 \\
\hline H012C54 & 81,53 & 23,6 & 2,59 & 4,3 & 0 & 0 & - & - \\
\hline H013C43 & 81,02 & 25,7 & 2,85 & 9,3 & 0 & 0 & 1,03 & 1,19 \\
\hline M014C50 & 83,34 & 28,3 & 3,32 & 10,8 & 0 & 0 & 0,94 & 1,222 \\
\hline H015C50 & 69,34 & 64 & 5,75 & 111,1 & 17 & 9,9 & 0,56 & 0,5 \\
\hline H016C55 & 67,3 & 75,7 & 16,62 & 57,5 & 5 & 5,2 & 0,75 & 0,96 \\
\hline H017C70 & 80,8 & 17,6 & 1,89 & 13,7 & 0 & 0 & - & - \\
\hline H018C40 & 80,41 & 18,4 & 2 & 9,9 & 0 & 0 & 0,83 & 1,4 \\
\hline M019C62 & 57,61 & 38,4 & 2,08 & 25,6 & 1 & 1 & 0,69 & 1,327 \\
\hline Promedio x SD & $74,38 \pm 8,9$ & $40,37 \pm 30,3$ & $4,67 \pm 4,5$ & $31,94 \pm 37,33$ & $13,47 \pm 36,8$ & $2,64 \pm 6,0$ & $0,959 \pm 0,325$ & $1,102 \pm 0,385$ \\
\hline
\end{tabular}

Tabla 2. Parámetros de HRV en pacientes control.

\begin{tabular}{|l|c|c|c|c|c|c|c|c|}
\hline \multicolumn{1}{|c|}{ CÓDIGO } & FC (LPM) & SD RR (ms) & SD FC & RMSSD (ms) & NN50 (L) & pNN50 (\%) & ENT. APROX & $\begin{array}{c}\text { ENT. } \\
\text { MUESTRAL }\end{array}$ \\
\hline M001-43 & 62,72 & 55,3 & 3,77 & 37,2 & 117 & 15,6 & 1,43 & 1,64 \\
\hline H002-21 & 69,25 & 63,4 & 5,21 & 36,4 & 96 & 13,9 & 1,24 & 1,33 \\
\hline H003-21 & 74,8 & 69,2 & 6,46 & 40,1 & 139 & 18,6 & 1,1 & 1,18 \\
\hline H004-22 & 71,72 & 61,3 & 4,96 & 25,2 & 35 & 4,8 & 1,08 & 1,1 \\
\hline H005-24 & 83,31 & 38,4 & 4,48 & 20,9 & 16 & 1,9 & 1,29 & 1,41 \\
\hline H006-23 & 52,47 & 159,5 & 10,18 & 102,6 & 246 & 48,2 & 1,26 & 1,37 \\
\hline H007-57 & 69,99 & 53,4 & 4,37 & 26,4 & 38 & 5,5 & 1,21 & 1,32 \\
\hline 008-22 & 68,06 & 75,1 & 5,85 & 61,3 & 43 & 20,58 & 1,41 & 1,72 \\
\hline H009-24 & 85,39 & 31,2 & 3,66 & 15,3 & 3 & 0,4 & 1,23 & 1,28 \\
\hline H010-53 & 64,61 & 25,6 & 1,82 & 12,1 & 1 & 0,2 & 1,19 & 1,32 \\
\hline H011-42 & 72,5 & 37,1 & 3,23 & 28,4 & 60 & 8,1 & 1,43 & 1,74 \\
\hline H012-43 & 63,17 & 77,6 & 5,3 & 32,2 & 67 & 10,5 & 1,28 & 1,15 \\
\hline H013-51 & 62,15 & 44 & 4,61 & 28,9 & 5 & 0,7 & 1,065 & 1,033 \\
\hline H014-44 & 74,24 & 44,6 & 4,14 & 34,8 & 121 & 16,2 & 1,38 & 1,61 \\
\hline H015-15 & 85,93 & 46,9 & 5,51 & 23,3 & 27 & 3,1 & 1,12 & 1,13 \\
\hline H016.42 & 89,97 & 35,3 & 4,76 & 16,1 & 8 & 0,9 & 1,1 & 1,06 \\
\hline M017-22 & 103,54 & 29,5 & 5,04 & 14 & 8 & 0,8 & 1,08 & 0,99 \\
\hline M018-23 & 72,53 & 70 & 6,28 & 47,6 & 191 & 26,4 & 1,39 & 1,53 \\
\hline M019-21 & 64,01 & 51 & 3,65 & 49,2 & 232 & 36,4 & 1,44 & 1,88 \\
\hline Promedio x SD & $73,18 \pm 12,0$ & $56,23 \pm 29,6$ & $4,91 \pm 1,7$ & $34,32 \pm 21,01$ & $76,47 \pm 78,3$ & $12,3 \pm 13,3$ & $1,25 \pm 0,134$ & $1,358 \pm 0,269$ \\
\hline
\end{tabular}


Gutiérrez et al. (2009) analizaron la VFC de 36 pacientes chagásicos sin afectación cardiaca conocida, con valores de SDRR de $149,75 \pm 39,05 \mathrm{~ms}$, para pacientes y $156,53 \pm 41,84 \mathrm{~ms}$, para los controles, sin diferencias significativas entre grupos, resultados que, de acuerdo con lo establecido por De la Cruz et al. (2008), tendrían una variabilidad baja. Lo anterior, se podría atribuir a la edad de los pacientes del estudio, que promediaron los 36 años, edad temprana para que el parásito genere daño cardiaco, tal y como lo reportan Mitelman et al. (2013), quienes encontraron que los enfermos chagásicos crónicos tuvieron una edad significativamente mayor que los controles.

Variable RMSSD: Los valores de RMSSD para los dos grupos estudiados no arrojaron diferencias significativas, pero fueron claramente superiores en los controles que, en los pacientes, como era de esperarse, dado que el dato se deriva de la SDRR. Gutiérrez et al. (2009) encontraron valores de 39,31 $15,56 \mathrm{~ms}$, para controles

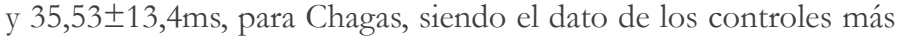
elevado que el de los positivos a Chagas, coincidiendo con nuestro hallazgo y con valores muy cercanos a los de este estudio. Ortiz \& Mendoza (2008) midieron en individuos sanos valores de RMSSD, con un promedio de $30,6 \mathrm{~ms}$, valor que se ubica muy cercano al obtenido en este estudio, en individuos seropositivos. Es probable que el resultado obedezca al número reducido de individuos analizados en este estudio. Rodas et al. (2008) reportaron valores promedio de esta variable de $64,0 \mathrm{~ms}$, en individuos considerados sanos. Los registros de este estudio, se hicieron en un tiempo de cinco minutos, lo cual, puede haber influenciado el resultado. En el estudio de Ortis et al. (2008), con individuos deportistas sanos, clasificados en dos grupos, de acuerdo con su calidad de vida, el RMSSD de los de calidad alta fue de $64,37 \pm 32,7 \mathrm{~ms}$ y para los

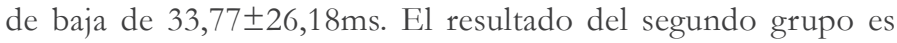
muy cercano al obtenido en este estudio para los controles, no deportistas. Concluyen que los individuos con mejor calidad de vida presentaron una mayor VFC, relacionada con una mayor actividad vagal en reposo. Gimenez et al. (2003) no encontraron diferencias significativas en el análisis de esta variable, además, consideraron valores normales aquellos que estén por encima de $27 \pm 12 \mathrm{~ms}$. Por el contrario, Mitelman et al. (2013) reportaron diferencias altamente significativas de la VFC en las variables de dominio temporal entre chagásicos y controles y afirman que los parámetros de VFC son conocidos y utilizados para evaluar la cardiopatía coronaria. Octavio et al. (2004) estudiaron los perfiles circadianos de la VFC en pacientes con Chagas crónico y reportaron valores

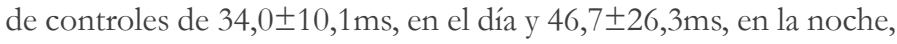
coincidiendo el reporte de las horas del día, con nuestros controles, cuyos registros se obtuvieron en horas de la mañana. El resultado lo explicaron como consecuencia de la disautonomía parasimpática que acompaña la enfermedad. Llama la atención de este estudio que en los pacientes controles, esta variable fue mayor en la noche, en donde predomina un mayor tono vagal. En el estudio de Ramos et al. (2014), la variable RMSSD valorada antes y después del ejercicio en pacientes chagásicos fluctuó entre 30-83ms, antes del ejercicio y 32-74ms, después del mismo, sin diferencias significativas entre los dos momentos, ni con respecto a los controles, resultado atípico, si se esperaba que la actividad física incrementara el tono vagal y la atribuyen, al igual que Octavio et al. (2004) y Merejo et al. 2018, a la disfunción autonómica, presencia de anticuerpos antimuscarinicos y daño del nodo sinusal, el cual, no responde a la capacidad del parasimpático de modular la variación de latido a latido de la frecuencia cardiaca.

Variables NN50 y pNN50: La NN50, para los controles, fue de $76,47 \pm 78,3$ latidos, mientras que para los seropositivos $13,47 \pm 36,8$ latidos, valores que tuvieron diferencias altamente significativas $(\mathrm{P} \leq$ 0,003), lo cual, indica una regularidad de intervalos RR más estrecha para los pacientes chagásicos, que corresponde con los valores de pNN50, que fueron $12,3 \pm 13,3 \%$ y $2,64 \pm 6,0 \%$, respectivamente, para el mismo orden de los grupos, con diferencias altamente significativas $(\mathrm{P} \leq 0,007)$.

Un comportamiento de la FC con NN50 y pNN50 en mayor número y proporción, implica una mayor variabilidad de la misma, hecho que es coherente con el funcionamiento de un corazón de persona sana, dado que refleja un mayor equilibrio entre el control simpático y parasimpático de la actividad cardiaca.

Gutiérrez et al. (2009) reportaron una pNN50 de 13,56士10,18\%,

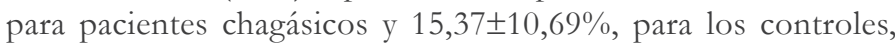
sin diferencias significativas, observándose más homogeneidad de resultado entre los grupos que el obtenido en este estudio. Probablemente, la diferencia radica en la edad promedio, que fue de 36 años. Para Gimenez et al. (2003), pNN50 mayores al 15\%, son considerados de alta variabilidad, mientras que resultados menores al 4\%, son clasificados como de baja variabilidad. De acuerdo con este concepto, las pacientes chagasicos de este estudio, muestran una variabilidad muy baja, situación que, en forma semejante a los resultados de las otras variables del dominio temporal de la VFC, se considera como consecuencia de la disfunción autonómica en el control cardiaco y reducción de los parámetros de modulación vagal e incremento de los de modulación simpática, con predominio de la alteración vagal (Merejo et al. 2018). Octavio et al. (2004) reportaron una pNN50 de 24 hs en controles, de $14,5 \pm 10,8 \%$ y $13,2 \pm 9,9 \%$, en Chagas, resultado cercano en los controles de este reporte, pero muy alejado para el caso de los seropositivos, lo cual, podría ser consecuencia del grado de afectación cardiaca de los pacientes involucrados en los dos estudios. La mayoría de los individuos que participaron en este trabajo son crónicos (edad promedio 52 años), con años de padecimiento de la enfermedad. Rodas et al. (2008) reportaron valores de pNN50 de 46\%, en individuos jóvenes y sanos, valor superior al encontrado en este estudio, probablemente, por el tiempo de registro, que fue de 5 minutos. Ortis et al. (2008) analizaron esta variable en individuos sanos jóvenes de dos condiciones físicas. Los de baja tuvieron un pNN50 de $6,11 \pm 7,97 \%$, mientras que los de alta registraron $16,4 \pm 9,96 \%$, resultado cercano al valor de nuestros controles.

Variables No Lineales: ApEn - SampEn: Las variables de medidas no lineales arrojaron para ApEn valores de 1,25 $\pm 0,134$, para los controles y de $0,959 \pm 0,325$, para los seropositivos, con diferencias altamente significativas ( $\mathrm{P} \leq 0,001)$, entre ellos. Para la SampEn los valores fueron 1,358 $\pm 0,264$ y 1,102 $\pm 0,385$, respectivamente, para 
controles y chagásicos, también con diferencias significativas $(\mathrm{P}$ $\leq 0,02)$.

Estas variables analizan el comportamiento caótico de la VFC, ya que la entropía, especifica la aleatoriedad y predicción de los sistemas (Guillén et al. 2003; Sady \& Ribeiro, 2016); la aproximada hace un cálculo rápido de la regularidad de las señales (Pincus et al. 1991). La muestral cuantifica la predicción de valores siguientes de una serie temporal basada en el conocimiento de los valores previos de la serie (Carrasco et al. 1994). En este estudio ambas entropías fueron significativamente más bajas para los pacientes chagásicos. Guillén et al. (2003) reportaron una entropía aproximada de $0,42 \pm 0,1$, para los controles y $0,34 \pm 0,15$, para pacientes chagásicos, con diferencias altamente significativas $(\mathrm{P}=0,002)$, valores que no coinciden con los nuestros, pero que concuerdan en la significancia estadística del resultado. Los resultados del estudio citado, se obtuvieron de pacientes en estado agudo de la enfermedad, mientras que los nuestros, corresponden a pacientes crónicos. En ambos estudios, el presentar una entropía aproximada mayor en los controles, indica una mayor irregularidad de la VFC y como se ha citado antes, es reflejo de un mejor estado de salud. Otra característica del estudio en mención es que arrojó un mayor número de ocurrencias en el grupo de chagásicos agudos que en el control, lo que significa incremento de regularidad de la variabilidad del ritmo cardíaco en los enfermos. Los métodos no lineales son usados para evaluar el balance del sistema nervioso autónomo sobre el corazón de pacientes chagásicos agudos y crónicos. La evaluación de la actividad autonómica cardiovascular puede ser útil para identificar, de manera temprana, sujetos que, eventualmente, pueden desarrollar una etapa más progresiva de la enfermedad (Rosas et al. 2007; 2002). Para Sady \& Ribeiro (2016), las variables de la entropía son capaces de detectar patrones selectivos en la regulación autonómica cardiovascular, que pueden predecir la muerte en pacientes con enfermedad de Chagas.

Se puede concluir, que el análisis de las variables de la VFC en los dominios de tiempo y No lineales, indican que, en pacientes con enfermedad de Chagas, con características, como los incluidas en este estudio, se presenta afectación de las variables del dominio de tiempo, en donde la SDRR fue más reducida en pacientes seropositivos a Chagas y la RMSSD mayor en los controles. Las variables NN50 y pNN50 presentaron diferencias altamente significativas, que resultan en valores más bajos para los seropositivos. El análisis de las variables no lineales de la VFC, arrojó que, tanto la entropía aproximada como la muestral, fueron significativamente más bajas en pacientes chagásicos. El resultado implica una mayor irregularidad de la VFC en los controles que, a su vez, es reflejo de un mejor estado de salud.

Agradecimientos: Los autores agradecen a la Dirección General de Investigaciones de la Universidad de los Llanos por el apoyo para el desarrollo de esta investigación; al Dr. Omar Ramírez Gómez, coordinador de Vigilancia en Salud del Departamento del Meta y cada uno de los pacientes, quienes en forma voluntaria y desinteresada colaboraron con la presente investigación. Conflictos de intereses: El manuscrito fue preparado y revisado con la participación de los tres autores, quienes declaramos que no existe ningún conflicto de intereses que ponga en riesgo la validez de estos resultados.

\section{REFERENCIAS}

1. ÁlVAREZ, I.; CRUZ, L. 2011. Fisiología cardiovascular aplicada en caninos con insuficiencia cardiaca. Rev. Medicina Veterinaria (Colombia). 1(21):115-132.

2. BAR-YAM, Y. 1997. Dynamics of complex systems. Addison Wesley (Reading, MA, United States). Disponible desde Internet en:

https:// fernandonogueiracosta.files.wordpress. com/2015/08/yaneer-bar-yam-dynamics-of-complexsystems.pdf (con acceso 01/09/2018).

3. BELERENIAN, G.; MUCHA, C.; CAMACHO, A. 2001. Afecciones cardiovasculares en pequeños animales. Intermédica (Buenos Aires, Argentina). 3-17p.

4. BOGUCKI, S.; NOSCZCZYK-NOWAK, A. 2015. Short term heart rate variability (HRV) in healthy dogs. Polish J. Veterinary Science (Polonia). 18(2):307-312. https://doi.org/10.1515/pjvs-2015-0040

5. BUZZANO, O.; MERCADO, M.; LIGHTOWLER, C.; PÉREZ, E.; MALDANA, H. 2012. Predicción de fenómenos arrítmicos a través de la variabilidad de la frecuencia cardiaca en caninos. Cardiología canina, Rev. Veterinaria (Argentina). 23(1):55-58.

6. CARRASCO, H.; PARADA, H.; GUERRERO, L.; DUQUE, M. 1994. Prognostic implications of clinical, electrocardiographic and hemodynamic findings in chronic Chagas' disease. Int. J. Cardiol (Holanda). 43:27-38. https://doi.org/10.1016/0167-5273(94)90087-6

7. DE LA CRUZ, B.; LOPEZ, C.; NARANJO, J. 2008. Analysis of heart rate variability at rest and during aerobic exercise: A study in healthy people and cardiac patients. Br. J. Sport Medicine (England). 42(9):715-720. https://doi.org/ 10.1136/bjsm.2007.043646

8. FORTES, H.; DE ALMEIDA, R.; SILVEIRA, D.; LLAGUNO, M.; RESENDE, L.; DIAS DA SILVA, V.; CORREIA, D. 2018. Cardiac autonomic modulation and long-term use of amiodarone in patients with chronic Chagasic cardiopathy. Pacing and Clinical Electrophysiology. 41(7): 788-798. https://doi.org/10.1111/pace.13384

9. GIMENEZ, L.; MITELMAN, J.; GONZALEZ, C.; BORDA, L.; BORDA, S. 2003. Anticuerpos antirreceptores autonómicos, alteraciones de la variabilidad de la frecuencia cardíaca y arritmias en sujetos con enfermedad de Chagas. Rev. Argentina de Card (Argentina). 71(2):109-113. 
10. GUHL, F. 1998. Estado actual del control de la enfermedad de Chagas en Colombia. En: Guhl, F.; Jaramillo, C. (eds). Curso-taller control de tripanosomiasis americana y leishmaniasis: aspectos biológicos, genéticos y moleculares. Corcas Editores (Bogotá, Colombia). p.47-81.

11. GUILlÉN, P.; JUGO, D.; CARRASCO, H.; ROJAS, R. 2003. Dinámica no lineal para caracterizar la variabilidad del ritmo cardíaco en pacientes chagásicos agudos. Scientific J. from the Experimental Faculty of Sciences (Venezuela). 11(4):276-283.

12. GUTIÉRREZ, O.; RAMÍREZ, M.; BARBOZA, M.; CALVO, N. 2009. Variabilidad de la frecuencia cardiaca en pacientes con enfermedad de Chagas en fase indeterminada. Reporte preliminar. Rev. Costarric. Cardiol (San José, Costa Rica). 11(2).

13. INSTITUTO NACIONAL DE SALUD, INS.; ORGANIZACIÓN PANAMERICANA DE LA SALUD, OPS. 2009. Guía Protocolo para la vigilancia en salud pública de Chagas. Disponible desde internet en: http:/ /www.sanandres.gov.co/documentos/salud/ GUIAS_CLINICAS_DE_ETV/Protocolo_Chagas.pdf (con acceso el 02/07/2018).

14. KHOR, K.; SHIELS, I.; CAMPBELL, F.; GREEER, R.; ROSE, A.; MILLS, P. 2014. Evaluation of a technique to measure heart rate variability in anaesthetized cats. The Veterinary J (Inglaterra). 199(2):229-235. https://doi.org/10.1016/j.tvjl.2013.11.006

15. MEREJO, C.; REIS, M.; PEREIRA, B.; PEDROSA, R. 2018. Dysautonomy in different death risk groups (Rassi score) in patients with Chagas heart disease. Pacing and Clinical Electrophysiology (Reino Unido). 41(3):238-245.

16. MITELMAN, J.; GIMENEZ, L.; ACUÑA, L.; GONZÁLEZ, C. 2013. Valorar la asociación de pruebas diagnósticas incruentas para la detección de alteraciones funcionales miocárdicas autonómicas y endoteliales en la Enfermedad de Chagas. Rev. Guatemalteca de Cardiología. 23(1):16-20.

17. MITELMAN, J.; GIMENEZ, L.; ACUÑA, L.; TOMASELLA, M. 2015. Estudio por presurometría y Holter de los trastornos disautonómicos en la enfermedad de chagas. Rev. Guatemalteca de Cardiología (Guatemala). 25(1):1116.

18. MORA, M.; BARRIO, A.; NASSER, J.; SÁNCHEZ, O.; MARCO, D.; GONORAZKI, J. 1998. Contribution of the molecular biology technics at Chagas $\square$ disease. Bol Acad Nac Med (Argentina). 76(1):103-108.

19. OCtavio, A.; ROdrÍguez, A.; MisticCHiO, F.; MARCANO, F.; JIMÉNEZ, J.; MOLEIRO, F. 2004. Perfiles circadianos de la frecuencia cardíaca y de su variabilidad instantánea en una población de pacientes con infección chagásica crónica. Rev. Esp. Cardiología (España). 57(2):99189.

https://doi.org/10.1016/S0300-8932(04)77075-X

20. ORTIS, L.; RODAS, G.; OCAÑA, M.; PARRADO, E.; PINTANEL, M.; VALERO, M. 2008. Variabilidad de la frecuencia cardíaca como indicador de salud en el deporte: Validación con un cuestionario de calidad de vida (SF-12) Apunts Medicine de l'Esport (España). 43(158):62-69. https://doi.org/10.1016/S1886-6581(08)70073-2

21. ORTIZ, J.; MENDOZA, D. 2008. Variabilidad de la frecuencia cardiaca, una herramienta útil. Efdeportes (Argentina). 13(121).

22. PAN AMERICAN HEALTH ORGANIZATION (PAHO), WORLD HEALTH ORGANIZATION, (WHO). 2017. General Information-Chagas Disease. Disponible desde internet en:

http://www.paho.org/hg/index.php?option=com topics\&view $=$ article $\& i d=10 \& l$ temid $(\operatorname{con}$ acceso el 09/07/2017).

23. PINCUS, S.; GLADSTONE, I.; EHRENKRANZ, R. 1991. A regularity statistic for medical data analysis. J. Clin. Monit (United States). 7(4):335-345.

24. RAMOS, B.; OLIVEIRA, M.; PEREIRA, M.; NOMAN, M.; SILVEIRA, H.; PINTO, M.; SERAFIM, V.; DA COSTA, M.; PINHO, A. 2014. Effects of Exercise Training on Heart Rate Variability in Chagas Heart Disease. Arq. Bras. Cardiol (Brasil). 103(3):201-208. https://doi.org/10.5935/abc.20140108

25. RASMUSSEN, C.; FALK, T.; DOMANJKO, A.; SCHALDEMOSE, M.; ZOIS, N.; MOESGAARD, S.; ABLAD, B.; NILSEN, H.; LJUNGVALL, L.; HOGLUND, K.; HAgGSTROM, J.; PEDERSEN, H.; BLAND, J.; OLSEN, L. 2014. Holter monitoring of small breed dogs with advanced myxomatous mitral valve disease with and without a history of syncope. J. Veterinary Internal Medicine (Egypt). 28(2):363-370. https://doi.org/10.1111/jvim.12290

26. RICHMAN, J.; MOORMAN, J. 2000. Physiological time-series analysis using approximate entropy and sample entropy. American J. Physiology-Heart and Circulatory Physiology (United States). 278(6):2039-2049. https://doi.org/10.1152/ajpheart.2000.278.6.H2039

27. RODAS, G.; CARBALLIDO, C.; RAMOS, J.; CAPDEVILA, L. 2008. Variabilidad de la frecuencia cardiaca: concepto, medidas y relación con aspectos clínicos. Archivos de Medicina del Deporte (España). 25(123):41-47. 
28. ROSAS, F.; GUHL, F.; VELASCO, V.; NICHOLLS, R.; VILLEGAS, F.; BEANCOURT, J.; MELGAREJO, I. 2007. Cardiomiopatía Chagásica. En: Rosas, F; Vanegas, D.; Cabrales, M. (eds). Enfermedad de Chagas, $1^{\text {a }}$ Ed. Sociedad Colombiana de Cardiología (Bogotá, Colombia). p.47-62.

29. ROSAS, F.; VELASCO, V.; JUMBO, L.; RODRÍGUEZ, D.; ARBOLEDA, F.; JARAMILLO, C. 2002. Cardiomiopatía de Chagas. Acta Med. Colomb (Colombia). 27:26-40.

30. SADY, C.; RIBEIRO, A. 2016. Symbolic features and classification via support vector machine for predicting death in patients with Chagas disease. Computers in Biology and Medicine (Reino Unido). 70:220-227
31. SÁNCHEZ, J.; PARRADO, E.; CAPDEVILA, L. 2013. Variabilidad de la frecuencia cardiaca y perfiles psicológicos en deportes de equipo de alto rendimiento. Rev. Psicología del Deporte (España). 22(2):345-352.

32. TUNDO, F.; LOMBARDI, F.; ROCHA, M.; BOTONI, F.; SCHMIDT, G.; BARROS, V. 2005. Heart rate turbulence and left ventricular ejection fraction in Chagas disease. EP Europace (England). 7(3):197-203. 